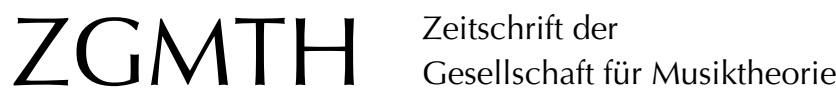

Schiltz, Katelijne (2013): Christiane Wiesenfeldt, Majestas Mariae. Studien zu marianischen Choralordinarien des 16. Jahrhunderts (= Beihefte zum Archiv für Musikwissenschaft 70), Stuttgart: Franz Steiner Verlag 2012. ZGMTH 10/1, 207-210.

https://doi.org/10.31751/716

(C) 2013 Katelijne Schiltz

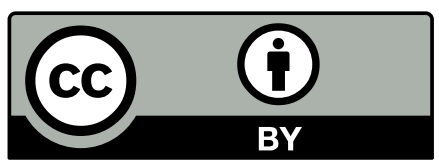

Dieser Text erscheint im Open Access und ist lizenziert unter einer Creative Commons Namensnennung 4.0 International Lizenz.

This is an open access article licensed under a

Creative Commons Attribution 4.0 International License.

veröffentlicht / first published: 06/07/2014

zuletzt geändert / last updated: 19/02/2016 


\section{Christiane Wiesenfeldt, Majestas Mariae. Studien zu marianischen Choralordinarien des 16. Jahrhunderts (= Beihefte zum Archiv für Musikwissenschaft 70), Stuttgart: Franz Steiner Verlag 2012}

Die Aussage mag zunächst überraschen: Die etwa 70 im Zeitraum von ca. 1490 bis ca. 1630 überlieferten Missae de Beata Virgine (im Folgenden: BV) stellen die umfangreichste 'Messgattung، der Frühen Neuzeit dar - ihre Zahl übertrifft zum Beispiel bei weitem die heute viel bekanntere Messengruppe zum L'homme armé. Das Besondere ist, dass den Ordinariumsteilen keine gemeinsame Vorlage - sei es nun eine geistliche oder weltliche Melodie -, die sich als roter Faden die gesamte Messe zieht, zugrunde liegt, sondern dass jeder Teil eine eigene Choralvorlage hat. Da dies nicht so recht zur Zyklusidee, die man in der Musikwissenschaft traditionell von der Messe aus dieser Zeit hat, passt, wurde dieser Messentyp in der Forschung häufig mit zweckmäßiger Gelegenheitsmusik in Verbindung gebracht, eher herablassend betrachtet und fand meistens nur am Rande Beachtung. Dass die Missae de Beata Virgine in vielerlei Hinsicht (musikalisch, theologisch, wissenschaftsgeschichtlich usw.) ein faszinierender Forschungsgegenstand sind, zeigt nun Christiane Wiesenfeldt, die sich in ihrer an der Universität Münster entstandenen Habilitationsschrift diesem Thema gewidmet und dazu ein eindrucksvolles Buch vorgelegt hat.

Im Anschluss an ein Kapitel zum Forschungsgegenstand und zur Methode widmet sich die Autorin ausführlich den Geschichtsbildern der polyphonen Messe. In einem ersten Abschnitt setzt sie sich - anknüpfend an Andrew Kirkmans Untersuchung zur Entstehung der zyklischen Messe ${ }^{1}$ - kritisch mit dem Zyklusbegriff, der die Musikgeschichtsschreibung vor allem seit dem 19. Jahrhundert prägt,

$1 \quad$ Kirkman 2001. auseinander. In weiteren Abschnitten thematisiert sie die Idee der »Funktion als Beschränkung" und des »emanzipierten Komponisten" (im Sinne eines von funktionalen Ansprüchen freien Künstlers) - zwei nicht unproblematische Konzepte, die für das Thema der Missae de Beata Virgine besondere Relevanz haben.

Auf diese methodischen und terminologischen Kapitel, die ungefähr ein Fünftel des Buchs ausmachen, folgt eines über »Maria als künstlerisches und religiöses Motiv bis 1600 «. Ein erster Teil beleuchtet ekklesiologische Grundlagen zu Begriffen wie "Mater Christi«, "Mater credentium " und "Mater ecclesia". Insbesondere Marias Rolle als smediatrixı, die nicht zuletzt auf ihre paradoxe Gestalt (Jungfrau und Mutter) zurückgeht, erweist sich auch im musikalischen Umgang - vor allem im Gloria-Tropus Spiritus et alme - als ein zentrales Motiv.

Der Abschnitt »Medien des Marienlobs« ist marianischen Manifestationen in der Liturgie, in Gebeten und Festen einerseits und in Literatur, Kunst und Musik andererseits gewidmet. Obwohl der Überblick keinen Anspruch auf Exhaustivität erhebt, hätten noch weitere Aspekte eine Erwähnung verdient. Beispielsweise sei auf Phänomene wie die gelegentliche ,Politisierung، von Marienfesten verwiesen: Wenn die Stadt Venedig ihr Gründungsfest auf den 25. März (Mariä Empfängnis) legt und ihren Entstehungsmythos somit liturgisch verankert, dient die Betonung des sunbefleckten Zustands (vgl. auch das berühmte Gedicht von Thomas Coryat aus dem Jahr 1611, in dem die Stadt mit einer "pure Virgin and incontaminated mayde " verglichen wird) nicht zuletzt der politischen Selbstdarstellung. Ein weiterer Punkt, der auch musikalisch relevant 
ist, ist die der Jungfrau zugeschriebene heilende Kraft. So wurde etwa das Lied Maria zart, das um 1500 besondere Verbreitung fand, sowohl im Hinblick auf die যJenseitsvorsorge auch zum Schutz vor Syphilis gesungen. ${ }^{2}$

Ab Kapitel $V$ werden dann Messvertonungen vom späten 15 . bis zum 17. Jahrhundert aus historischer, quellenkritisch-kodikologischer und analytischer Perspektive untersucht. Dieser Abschnitt ist sicher das Kernstück des Buchs. Dabei reichen die geographischen Schwerpunkte von Habsburg-Burgund über Mantua und Rom bis nach Spanien und Portugal. Wiesenfeldt gelingt es, ein dichtes Netzwerk an kompositorischen Bezügen deutlich zu machen und dieses differenziert und kontextabhängig zu untersuchen. In einem ersten Abschnitt wird Pierre de la Rues von der Musikwissenschaft postulierte Rolle als Katalysator der ganzen BV-Tradition kritisch beleuchtet. Zwar schuf er die früheste zusammenhängend überlieferte Missa de Beata Virgine - eine Tatsache, die zweifellos mit der Marienfrömmigkeit seiner Mäzenin Margarete von Österreich in Verbindung steht -, aber die Komposition weist eine singuläre Auswahl von Vorlagen auf. Während La Rues Messe den Repräsentationswillen Margaretes widerspiegelt, sind Heinrich Isaacs BV-Messen - es sind vier bis sechs Vertonungen von ihm überliefert (die Forschung ist sich über die genaue Zahl nicht einig, Wiesenfeldt liefert aber überzeugende Argumente für die Zuschreibung der vierstimmigen Messe) - im privaten Kontext des kaiserlichen Hofes einzuordnen (118); dafür spricht auch ihre ausschließlich handschriftliche Überlieferung. Bemerkenswert sind hier vor allem die zahlreichen formalen und motivischen Beziehungen der Messen untereinander.

Im folgenden Abschnitt wird der Fokus nach Rom verlagert. Die große Bedeutung des marianischen Tropus für das Selbstverständnis der Päpste ist eine Erklärung für die hohe Zahl von zehn BV-Messen, die in vatikanischen Quellen bis etwa 1539 vorliegen. Dazu gehören La Rues bereits genannte Messe, aber auch

2 Vgl. Lodes 2001.
Vertonungen von Brumel, Josquin, Morales und von heutzutage weniger bekannten Komponisten wie Michot, Misonne und d'Argentil. Scheint dies zunächst auf eine geschlossene Werkgruppe hinzuweisen, so fallen gleichzeitig die gravierenden Unterschiede zwischen diesen Messen ins Auge, nicht zuletzt hinsichtlich der Wahl der Choralvorlage.

Im Falle Palestrinas zeigt Wiesenfeldt, dass die vierstimmige BV-Messe aus dem Missarum liber secundus von 1567 - anders als es in der Forschung meist vertreten wird - sehr wohl mit dem Gloria-Tropus Spiritus et alme komponiert wurde; in dem römischen Exemplar wurden allerdings die entsprechenden Stellen getilgt (165ff.). Weiterhin stellt sich heraus, dass seine beiden BV-Messen (für vier und sechs Stimmen) mit ihrer oft problematischen Diktion und den sehr dichten Texturen nicht so richtig in das traditionelle Palestrina-Bild passen wollen. Eine Ausnahme stellt u. a. eine Passage im sechsstimmigen Gloria dar: Mit der homophonen Deklamation von »Mariam (coronans) « stellt Palestrina außerdem einen intertextuellen Bezug zum Gloria aus Josquins gleichnamiger Messe her. Dies sollte aber nicht darüber hinwegtäuschen, dass in Palestrinas Vertonungen der Bezug zu Cristóbal de Morales' BV-Messen, die auch untereinander sehr viele Verbindungen (Moduswahl, Vorlagen, Motive usw.) aufweisen, viel stärker ist.

Mit diesem Beispiel schlägt die Autorin den Bogen zu Spanien. Am Anfang dieses Abschnitts steht eine bemerkenswerte Gruppe von Kompilationsmessen, die in Handschriften zwischen ca. 1500 und 1520 überliefert sind und an denen zahlreiche namentlich bekannte und anonyme Komponisten beteiligt waren. Auffällig ist hier die dezidiert spanische Orientierung in der Auswahl der Komponisten. Weiterhin hervorzuheben sind die zahlreichen «Werkpaare : Sowohl Morales als auch Francisco Guerrero und Sebastián de Vivanco schufen jeweils zwei BV-Messen - was im römischen Repertoire sonst nur bei Palestrina der Fall ist.

Mit einem Abschnitt zu drei Chorbüchern aus Aachen, in denen insgesamt vier Marienmessen überliefert sind, lenkt Wiesenfeldt den 
Blick auf eine Region, die durch ihre geographische Lage im Grenzgebiet der deutschen Konfessionskriege große Bedeutung erlangte. Die Autorin richtet ihr Augenmerk insbesondere auf die Tropusbehandlung bei den Komponisten Johannes Mangon und Pierre Chenemont. Wenn sie aber versucht, anhand eines Stilvergleichs - plakativ ausgedrückt sschlichts versus spolyphon nologie der Messen Mangons herzustellen (229-231), so muss dagegengehalten werden, dass ein schlichter Stil nicht unbedingt auf eine frühere Datierung hinweisen muss - eine Tatsache, die Wiesenfeldt selbst in ihrem Abschnitt zu Morales' Messen betont.

Ein weiterer Abschnitt ist dem Komplex von Mantuaner Messen gewidmet. Sie entstanden alle für die Santa Barbara-Liturgie, die eine eigene Zeremonialordnung hatte. Unter dem Stichwort »Montagen" stellt die Autorin interessante Überlegungen zu einer Reihe von Eingriffen an, die Komponisten vornahmen, um bereits existierende Stücke konform mit der Mantuaner Liturgie zu gestalten. Wichtig ist hier vor allem das Gloria, das in den 1560er Jahren noch tropiert werden durfte, dessen Tropus ab 1570 hingegen entfernt wurde. Während Palestrina also im Auftrag von Guglielmo Gonzaga in den Jahren 1578-1579 nicht weniger als drei BV-Messen schrieb, in denen der Gloria-Tropus bereits fehlt, wurde der Kapellmeister Giaches de Wert damit beauftragt, frühere Marienmessen, die den Tropus noch enthielten, neu zu arrangieren und die entsprechenden Passagen zu entfernen. Dies illustriert Wiesenfeldt eindrucksvoll anhand des Gloria aus Giovanni Continos BVMesse, dessen Charakter sich am Ende sogar von einem ruhigen Amen in einen rhythmisch lebhaften Schlussgestus verändert (240).

Im letzten Abschnitt, der den Titel ")Gattungsdämmerung، in Portugal? « trägt, zeigt die Autorin anhand von BV-Messen aus dem 17. Jahrhundert, dass portugiesische Komponis- ten wie etwa Duarte Lobo, Manuel Cardoso und Filipe de Magalhães in ihren BV-Messen versuchten, eine neue Sprache zu entwickeln, die zugleich zur nationalen Identitätsbildung beitrug und dafür sorgte, dass ihre Messen bis ins 19. Jahrhundert populär blieben, und das sogar in Latein-Amerika. Zwar spricht aus der kontrapunktischen Textur dieser Messen einerseits eine Vertrautheit mit der Tradition - insbesondere mit den Messen von Morales und Guerrero -, doch weist andererseits die Hierarchisierung der Stimmverhältnisse auf ein monodisches Klangkonzept hin (250).

Ein systematisch-chronologisches Verzeichnis der Missae de Beata Virgine (260-281) informiert über die Entstehungszeit, Besetzung, formale Disposition (Choralvorlage der einzelnen Teile, Modus, tropiert/nicht tropiert) sowie Quellen und Editionen der jeweiligen Messen. Auch der Katalog von weiteren, einzeln überlieferten Gloriae de Beata Virgine (282-285) - oft ohne Komponistenzuschreibung - dürfte eine sehr nützliche Grundlage für weitere Forschungen werden.

Wiesenfeldts Buch beeindruckt durch die große Materialfülle, die detaillierten Analysen sowie die überzeugende Verbindung von historischen und musikwissenschaftlichen Untersuchungen, Quellenstudium und methodischen Überlegungen. Überzeugend macht sie deutlich, dass der BV-Komplex - anders als zumeist angenommen - für die Geschichte der Messe von großer Bedeutung ist. Die Marienthematik als »übergeordnetes Movens" (254) bildet ein sowohl quantitativ als auch qualitativ wichtiges Korpus an Messen, das von einer großen Dynamik - etwa im Umgang mit den marianischen Passagen oder in der Anpassung an lokale Traditionen - geprägt ist und uns somit dazu zwingt, unser Geschichtsbild der polyphonen Messe gründlich zu überdenken.

Katelijne Schiltz 


\section{Literatur}

Kirkman, Andrew (2001), »The Invention of the Cyclic Mass", Journal of the American Musicological Society 54/1, 1-47.

Lodes, Birgit (2001), »Maria zart und die Angst von Fegefeuer und Malafrantzos - Die Karriere eines Liedes zu Beginn des 16. Jahrhunderts", in: Musikalischer Alltag im 15. und 16. Jahrhundert, Kassel, 2001 (= Trossinger Jahrbuch für Renaissancemusik 1), hg. von Nicole Schwindt, 99-131. 